\title{
Experimental and Theoretical Investigation of
}

the Distance Dependence of Localised Surface

\section{Plasmon Coupled Förster Resonance Energy}

\section{Transfer}

Xia Zhang(1), Cristian A. Marocico(1), Manuela Lunz(1)†, Valerie A. Gerard (2), Yurii K. Gun'ko (2), Vladimir Lesnyak (3), Nikolai Gaponik (4), Andrei S. Susha (5), Andrey L. Rogach (5), and A. Louise Bradley (1)*

(1) School of Physics and CRANN, Trinity College, Dublin 2, Ireland

(2) School of Chemistry and CRANN, Trinity College, Dublin 2, Ireland

(3) Istituto Italiano di Tecnologia, Via Morego 30, 16163 Genova, Italy

(4) Physical Chemistry, TU Dresden, Bergstrasse 66b, 01062 Dresden, Germany

(5) Department of Physics and Materials Science \& Center for Functional Photonics (CFP), City University of Hong Kong, Hong Kong, China

\section{KEYWORDS}

Quantum dots, Nanocrystals, Colloidal metal nanoparticles, Localised surface plasmons, Förster resonant energy transfer, Non-radiative energy transfer 


\section{ABSTRACT}

The distance dependence of localised surface plasmon (LSP) coupled Förster resonance energy transfer (FRET) is experimentally and theoretically investigated using a trilayer structure comprised of separated monolayers of donor and acceptor quantum dots with an intermediate $\mathrm{Au}$ nanoparticle layer. The dependence of the energy transfer efficiency, rate and characteristic distance, as well as the enhancement of the acceptor emission, on the separations between the three constituent layers is examined. A $d^{-4}$ dependence of the energy transfer rate is observed for LSP coupled FRET between the donor and acceptor planes with the increased energy transfer range described by an enhanced Förster radius. The conventional FRET rate also follows a $d^{-4}$ dependence in this geometry. The conditions under which this distance dependence is valid for LSP coupled FRET are theoretically investigated. The influence of the placement of the intermediate Au NP is investigated and it is shown that donor-plasmon coupling has the greater influence on the characteristic energy transfer range in this LSP coupled FRET system. The LSP enhanced Förster radius is dependent on the $\mathrm{Au}$ nanoparticle concentration. The potential to tune the characteristic energy transfer distance has implications for applications in nanophotonic devices or sensors. 
Förster resonance energy transfer (FRET) is a well-defined distance dependent dipole-dipole interaction. ${ }^{1}$ It has been widely used for measuring the distance between two fluorophores. ${ }^{2-4}$ However, as a spectroscopic ruler conventional FRET suffers from a limited length scale of approximately $10 \mathrm{~nm}$. Plasmonic structures provide a route towards longer range non-radiative energy transfer (NRET) via nanometal surface energy transfer (NSET) or localised surface plasmon (LSP) coupled FRET. ${ }^{5,6}$ The near-field of the plasmonic structure can modify the emission of a fluorophore. ${ }^{7-}$ ${ }^{12}$ Depending on the plasmonic properties of the metallic nanoparticle (MNP) it can lead to quenching or enhancement of the fluorophore emission. The modified emission can be a consequence of many processes such as NRET to the MNP, scattering and absorption by the MNP, and changes in the emitter's radiative and nonradiative decay rates. The plasmonic nanoparticle can also modify the excitation rate. NRET directly from fluorophores to MNPs has been demonstrated to provide a longer interaction range than traditional FRET pairs. ${ }^{5}$ The distance dependence of the energy transfer from dyes or light-emitting colloidal quantum dots (QDs) to MNPs has been described using the NSET formalism for single emitter-metallic nanosphere pairs ${ }^{13,14}$ and for planes of nanospheres. ${ }^{15}$

LSP coupled FRET between donor-acceptor pairs has also been theoretically investigated. ${ }^{16-18}$ Increases in the energy transfer rate, efficiency and range can be expected depending on the plasmonic properties of the MNP, and the relative positions of the donor and acceptor. With three constituent elements (namely donor, acceptor and plasmonic structure), LSP coupled FRET is a complex mechanism with a large number of parameters that can be tuned. The near-field of plasmonic nanostructures modifies the emission of the donor and acceptor and influences their near-field interaction through LSP coupled FRET. The FRET mechanism between the 
donor and acceptor competes with other decay mechanisms. Therefore, the various dependences need to be fully investigated and well understood to develop systems which can take advantage of plasmon controlled FRET for light emission, light harvesting or sensing applications.

Experiments have verified that enhancement of the energy transfer rate, efficiency, and range is achieved in different geometries with a variety of fluorophores and MNPs. ${ }^{6,19-29}$ LSP coupled FRET has been observed using single metallic nanoparticles coupled to a single donor-acceptor pair ${ }^{6}$, core-shell structures ${ }^{23-}$ ${ }^{25}$ and planar structures. ${ }^{26-28}$ Studying FRET from a conjugated polymer to fluorescent multilayer core-shell NP, Lessard-Viger et al. observed a 70\% increase in the Förster radius and an increase in the FRET-rate by two orders of magnitude. ${ }^{24}$ Also using a core-shell geometry, composed of donor and acceptor fluorophore molecules embedded in a shell coating a Au-Ag core-shell nanocrystal, Wang et al. demonstrated that FRET could be switched on and off by varying the spectral position of the LSP resonance relative to the donor emission and acceptor absorption. ${ }^{25}$ LSP coupled FRET was reported in planar structures comprised of a layer of dyes and/or QDs, acting as donors and acceptors, placed on a MNP layer. There is the possibility to enhance ${ }^{26}$ or inhibit ${ }^{29}$ the energy transfer process depending on how the LSP coupled FRET rate competes with the rates of the other processes. The competition between plasmon modified radiative rate, non-radiative rate and LSP coupled FRET rate is influenced by the spectral properties of the donor and acceptor, the overlap with the surface plasmon resonance and the relative placement of the donor and acceptor within the near-field of the plasmonic structure. The impact of modifying the plasmon field has been probed by tuning the MNP $\operatorname{size}^{28}$ and changing the distance between the donor-acceptor pair and a MNP layer. ${ }^{29}$ 
LSP enhanced FRET has also been observed in multilayer structures with separated donors, acceptors and plasmonic nanostructures..$^{27,30,31}$ The multilayer geometry provides the opportunity to study the dependence of LSP coupled FRET on the concentrations of the constituent layers as well as the separations between the layers and the ordering of the layers. The authors have previously reported that, by tuning the concentration of an intermediate Au NP monolayer, the LSP coupled FRET rate between monolayers of donor and acceptor QDs could be increased by a factor of $\sim 200$, with $\sim 150$-fold enhancement of the FRET efficiency and $240 \%$ increase of the Förster radius for the highest $\mathrm{Au}$ NP concentration investigated. ${ }^{30}$ It was also demonstrated that the maximum acceptor emission enhancement does not occur at the $\mathrm{Au}$ NP concentrations corresponding to the highest LSP coupled FRET rates. This is a consequence of competition between enhancement of the acceptor emission due LSP coupled FRET from the donor QDs and direct quenching of the acceptor emission via energy transfer to the MNP layer. ${ }^{30}$ The influence of preferential donor-plasmon coupling or acceptor-plasmon coupling on LSP mediated FRET was investigated by changing the order of the donor QD layers, acceptor QD layers and Au nanospheres layers in a multilayer stack. ${ }^{31}$

It is important to understand how LSP coupled FRET parameters, such as the energy transfer efficiency, rate and characteristic distance, depend on the separation between the donor and acceptor, in the presence of plasmonic nanostructures. In this work, trilayer structures with varying separations between the constituent layers are experimentally and theoretically investigated to determine how coupling to plasmonic nanostructures influences the distance dependence of LSP coupled FRET. Experimental measurements show evidence of strong donor-plasmon coupling and a dipole-dipole FRET-like distance dependence of the LSP coupled FRET is observed. 
It is found that the extended energy transfer range, in comparison with conventional FRET, can be described by an increase in the effective Förster radius in the presence of MNPs. A theoretical study is undertaken to further investigate the ranges over which the FRET-like distance dependence of the LSP coupled FRET mechanism is valid for this experimental system. The theoretical results also demonstrate that greater enhancement of the LSP coupled FRET efficiency is achieved for preferential donor-plasmon coupling compared with acceptor-plasmon coupling.

\section{RESULTS AND DISCUSSION}

Multilayer planar structures were assembled using a layer-by-layer (LbL) technique. $^{32}$ The trilayer structure is comprised of an acceptor CdTe QD monolayer, an intermediate Au NP monolayer and a donor CdTe QD monolayer, each separated by a polyelectrolye (PE) spacer layer. PE spacer layers have been previously used to investigate the distance dependence of NRET between separated QD monolayers and in QD-plasmon coupled systems. ${ }^{33-37}$ Donor-acceptor QD bilayers, QD-Au NP bilayers and QD monolayers were also prepared as reference samples. Schematics of the trilayer, donor-acceptor QD bilayer and QD-Au NP bilayer reference structures are shown in Figure 1(a).

The donor and acceptor QDs are negatively charged CdTe QDs stabilized by thiolglycolic acid in aqueous solution. ${ }^{38,39}$ The donor QDs, with a diameter of $2.6 \mathrm{~nm}$, have an emission peak at $547 \mathrm{~nm}$. The larger acceptor QDs, with a diameter of $3.4 \mathrm{~nm}$, have maximum emission at $660 \mathrm{~nm}$. Emission and absorption spectra for the donor and acceptor QD and Au NP monolayers are shown in Figure 1(b). As can been seen, this donor-acceptor QD pair has spectrally separated PL emission spectra. For 
measurement of the donor PL decays a $550 \mathrm{~nm}$ broadband filter with a full-widthhalf-maximum of approximately $(70 \pm 5) \mathrm{nm}$ was used to select the donor QD emission. The $5.5 \mathrm{~nm}$ diameter, colloidal Au NPs are positively charged. ${ }^{40}$ The $\mathrm{Au}$ NP and QD monolayer concentrations were determined from the absorption spectra. $^{38,41,42}$ The LSP resonance of the Au NP monolayer is clearly evident from the peak in the absorption at $\sim 532 \mathrm{~nm}$, which overlaps well with the donor emission peak, see Figure 1(b).
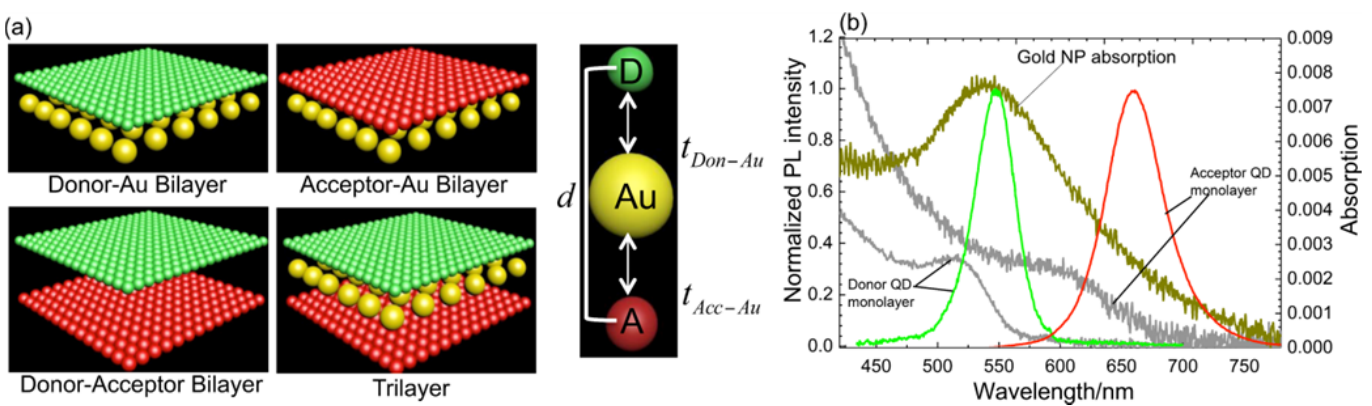

Figure 1. (a) Schematics of the trilayer, donor-acceptor bilayer and QD-Au NP bilayer

reference structures. The PE spacer layer thickness is denoted by $\mathrm{t}$, where $t_{D o n-A u}$ and $t_{A c c-A u}$ correspond to the PE spacer layer thickness between the intermediate Au NP monolayer and the donor QD and acceptor QD monolayers, respectively. The donoracceptor center-to-center distance is denoted by $d$. (b) Normalized PL spectra of a donor QD monolayer (green line) and acceptor QD monolayers (red line). Absorption spectra of both the QD monolayers (grey lines) and Au NP layer (yellow line) are also shown.

To establish that clear signatures of LSP coupled FRET are observed for this donor-acceptor pair, photoluminescence (PL) spectra of the trilayer structure as well 
as reference donor-acceptor QD and QD-Au NP bilayer samples are shown in Figure 2(a). Firstly, we consider the trilayer sample with $t_{D o n-A u}=3 \mathrm{~nm}$ and $t_{A c c-A u}=12 \mathrm{~nm}$. The donor and acceptor QD monolayers in the reference bilayer sample are separated by a PE spacer layer with thickness $t_{D o n-A c c}=21 \mathrm{~nm}$, which is equivalent to the surface-to-surface donor-acceptor separation in the trilayer structure. The samples have similar donor and acceptor QD concentrations, $c_{\text {Don }}=(2.8 \pm 0.2) \times 10^{17} \mathrm{~m}^{-2}$ and $c_{\text {Acc }}=(0.52 \pm 0.04) \times 10^{17} \mathrm{~m}^{-2}$, respectively. The Au NP concentration is $c_{A u}=(0.15 \pm 0.02) \times 10^{17} \mathrm{~m}^{-2}$. As can be seen in Figure 2(a), the acceptor emission in the trilayer sample is increased compared to the Au-absent donor-acceptor bilayer reference sample. The spectrum shows enhancement of the acceptor emission by a factor $\eta=\frac{I_{A c c, T r i}}{I_{A c c, B L}}=2.2$, where $I_{A c c, T r i}$ and $I_{A c c, B L}$ are the integrated acceptor PL in the trilayer structure and donor-acceptor bilayer reference sample, respectively. The donor and acceptor QD-Au NP bilayer structures show reduced emission relative to the donor-acceptor bilayer which is a consequence of the PL quenching effect of the 5.5. nm diameter Au NP layer. ${ }^{15,27}$ The PL quenching arises due to direct NRET from the QDs to the Au NPs. ${ }^{43}$ Therefore, the large acceptor enhancement in the trilayer structure is attributed to LSP coupled FRET from the donor to acceptor QDs.

Further evidence of LSP coupled FRET is seen on examination of the donor PL decays, shown in Figure 2(b). The donor PL decay shows a reduction of the average lifetime in the donor-Au NP bilayer structure, $\tau_{D o n, A u}=(2.8 \pm 0.2) \mathrm{ns}$, compared to the reference donor-acceptor bilayer structure, $\tau_{D o n, B L}=(3.7 \pm 0.2) \mathrm{ns}$. In the trilayer structure, the donor lifetime is further shortened to $\tau_{\text {Don,Tri }}=(2.24 \pm 0.06) \mathrm{ns}$ due to the introduction of the LSP coupled FRET mechanism from the donor QDs to the 
acceptor QDs. The LSP coupled FRET efficiency can be calculated using $E_{\text {LSP-FRET }}=1-\tau_{\text {Don, Tri }} / \tau_{\text {Don,Au }}$. An efficiency of $(20 \pm 7) \%$ is calculated for the example shown in Figure 2(b).
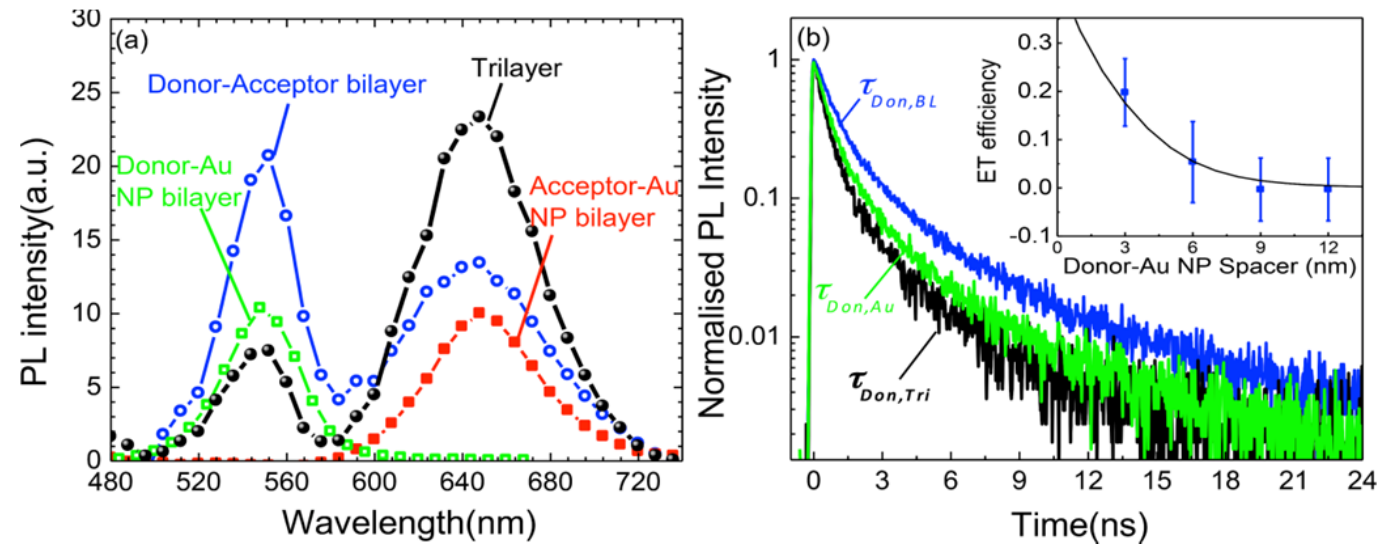

Figure 2. (a) PL spectra of the donor-acceptor bilayer with a $t_{D o n-A c c}=21 \mathrm{~nm}$ (blue open circle), donor-Au NP bilayer with $t_{D o n-A u}=3 \mathrm{~nm}$ (green open square) and the acceptor-Au NP bilayer with $t_{A c c-A u}=12 \mathrm{~nm}$ (red filled squares) which is subsequently capped with a PE spacer layer, $t_{D o n-A u}=3 \mathrm{~nm}$, and the donor QD monolayer to form the completed trilayer structure (black line, black spheres). All samples have $c_{\text {Don }}=(2.8 \pm 0.2) \times 10^{17} \mathrm{~m}^{-2} \quad$ and $\quad c_{A c c}=(0.48 \pm 0.08) \times 10^{17} \mathrm{~m}^{-2}$ respectively. The $\mathrm{Au} \mathrm{NP}$ concentration is $c_{A u}=(0.13 \pm 0.03) \times 10^{17} \mathrm{~m}^{-2}$.

Normalized donor PL decays for the donor-acceptor bilayer, labelled $\tau_{D o n, B L}$, donorAu NP bilayer with $t_{D o n-A u}=3 \mathrm{~nm}$, labelled $\tau_{D o n, A u}$, and the trilayer, labelled $\tau_{\text {Don,Tri }}$. The inset shows the LSP coupled FRET efficiency, $E_{L S P-F R E T}$, in the trilayer samples 
as a function of the donor-Au NP spacer layer thickness, $t_{D o n-A u}$. The result of the numerical simulation, calculated using equation 1 , is shown as a solid line.

To examine the dependence of the LSP coupled FRET signatures on the position of the Au NP monolayer, two distance dependent studies were undertaken. In the first case the acceptor QD-Au NP spacer layer thickness, $t_{A c c-A u}$, is constant while the donor QD-Au NP spacer layer thickness, $t_{D o n-A u}$, is varied. In the second case, $t_{D o n-A u}$ is fixed with varying $t_{A c c-A u}$. We consider firstly $t_{A c c-A u}$ fixed at $12 \mathrm{~nm}$ with $t_{D o n-A u}$ varying from $3 \mathrm{~nm}$ to $12 \mathrm{~nm}$. It was observed that the donor PL increases with increasing donor-Au NP separation and the acceptor PL rapidly reduces (PL spectra are given in the Supplementary Information, Figure S1). An increase in the donor PL is expected due to reduced direct quenching of the donor emission by NRET to the Au NPs as well as reduced LSP coupled energy transfer to the acceptors. Enhancement of the acceptor PL is observed only for the smallest donor-Au NP separation, after which the acceptor PL becomes dominated by the direct quenching by the Au NP layer. The influence of increasing $t_{D o n-A u}$ on the LSP coupled FRET efficiency, calculated from the donor PL decays, is shown in the inset of Figure 2(b). Determining the LSP coupled efficiency using $E_{\text {LSP-FRET }}=1-\tau_{\text {Don,Tri }} / \tau_{\text {Don,Au }}$ takes account of the effects of reduced direct energy transfer from the donor QDs to the Au NPs. The energy transfer efficiency decreases from $20 \%$ at $t_{D o n-A u}=3 \mathrm{~nm}$ to 0 for $t_{D o n-A u}=9 \mathrm{~nm}$.

We have calculated numerically the energy transfer rate and efficiency from the donor to acceptor QDs using a Green's tensor formalism. ${ }^{44}$ The Green's tensor of a $\mathrm{Au}$ nanosphere can be calculated from Mie theory, This can be extended to an 
ensemble of spheres through a multiple scattering method. ${ }^{45-47}$ The Green's tensor couples to dipolar sources, which can approximate fluorophores such as fluorescent dyes and QDs. Once the Green's tensor of the ensemble (in our case a monolayer of $\mathrm{Au}$ NPs) is known, the LSP coupled energy transfer rate, $k_{L S P-F R E T}$, is calculated directly from it via the following expression ${ }^{48}$

$k_{L S P-F R E T}=\tau_{D o n, A u}^{-1} 36 \pi^{2} Y_{D o n} \int_{0}^{\infty} d \lambda \lambda^{-2} f_{D o n}(\lambda) \sigma_{A c c}(\lambda)\left|\hat{n}_{D o n} \cdot G\left(\vec{r}_{D o n}, \vec{r}_{A c c}, \lambda\right) \cdot \hat{n}_{A c c}\right|^{2}$

where $\mathrm{G}$ is the Green's tensor of the system, $\mathrm{Y}_{\mathrm{Don}}$ is the intrinsic quantum yield of the donor, $f_{D o n}$ and $\sigma_{A c c}$ are the donor emission spectrum and acceptor absorption crosssection, respectively, and $\tau_{D o n, A u}$ is the calculated donor lifetime in the presence of the $\mathrm{Au}$ NP ensemble. ${ }^{49}$ The LSP coupled FRET efficiency, $E_{\text {LSP-FRET }}$, can then be obtained from

$$
E_{L S P-F R E T}=\frac{k_{L S P-F R E T}}{\tau_{D o n, A u}^{-1}+k_{L S P-F R E T}} .
$$

All the inputs to the model are taken from experimental values, such as the Au NP and acceptor QD concentrations, the donor QD emission spectrum, acceptor QD absorption spectrum, the donor quantum yield and spacer layer thicknesses. The measured quantum yield of the donor QD monolayer is 3\%. ${ }^{15,35}$ A correction term has been added to the dielectric permittivity of Au NPs to account for finite-size effects. We have fitted this term to the experimental absorption spectrum of Au NPs in solution.

To model layered structures, as in the experimental samples, we have calculated the energy transfer rate from a single donor to a layer of acceptors in the 
presence of the Au NP monolayer and we have averaged this energy transfer rate over a large number of realizations where the donor position in the donor monolayer has been randomly assigned. The acceptor and Au NP monolayers have been constructed by randomly placing individual acceptors and Au NPs in their respective monolayers, with the specific concentrations used in experiments. The results of the numerical simulation for the LSP coupled FRET efficiency, $E_{L S P-F R E T}$, as a function of $t_{D o n-A u}$ is shown as the solid line in the inset of Figure 2(b). There is good agreement between the theoretical and experimental results. It should be noted that the numerical model is not a fit, there are no free parameters, as discussed above.

Next we probe the dependence of the LSP coupled FRET efficiency on the acceptor-Au NP separation, $t_{A c c-A u}$. The donor-Au NP spacer layer thickness, $t_{D o n-A u}$, is fixed at $3 \mathrm{~nm}$, while $t_{A c c-A u}$ is varied from $3 \mathrm{~nm}$ to $27 \mathrm{~nm}$. The Au NP concentration is fixed at $c_{A u}=(0.12 \pm 0.01) \times 10^{17} \mathrm{~m}^{-2}$, with donor and acceptor QD concentrations of $\quad c_{D o n}=(1.8 \pm 0.2) \times 10^{17} \mathrm{~m}^{-2} \quad$ and $\quad c_{A c c}=(0.8 \pm 0.1) \times 10^{17} \mathrm{~m}^{-2}$ respectively. The donor PL decay lifetimes for the trilayer structures and a donor-Au NP bilayer (with fixed separation of $3 \mathrm{~nm}$ and the same Au NP concentration) are used to calculate the LSP coupled FRET efficiency and rate, $k_{\text {LSP-FRET }}=\tau_{D o n, T r i}^{-1}-\tau_{D o n, A u}^{-1}$, shown in Figure 3. The donor PL lifetime in the trilayer structure is strongly dependent on the acceptor-Au NP separation. The donor PL lifetime is shortest at the smallest acceptor-Au NP separations, which also corresponds to the smallest donor-acceptor separation, and steadily increases as the acceptor-Au NP separation increases. Correspondingly, the highest LSP coupled FRET efficiency and rate are observed at the shortest acceptor-Au NP separation. In 
Figure 3, the LSP coupled FRET efficiency, $E_{L S P-F R E T}$, is shown as a function of the acceptor-Au NP separation, $t_{A c c-A u}$, and the donor-acceptor center-to-center separation, $d=r_{D o n}+3 \mathrm{~nm}+2 \times r_{A u}+t_{A c c-A u}+r_{A c c}$, in which $r_{D o n}, r_{A c c}$ and $r_{A u}$ are the donor $\mathrm{QD}$, acceptor $\mathrm{QD}$ and $\mathrm{Au} \mathrm{NP}$ radii, respectively. An acceptor-Au NP separation, $t_{A c c-A u}$, ranging from $3 \mathrm{~nm}$ to $27 \mathrm{~nm}$ corresponds to donor-acceptor center-to-center distances, $d$, varying from $14.5 \mathrm{~nm}$ to $38.5 \mathrm{~nm}$.

To see the influence of the LSP coupling on the donor-acceptor energy transfer range, this data is compared with the NRET efficiency expected for a donoracceptor bilayers and donor-Au NP bilayers with the same center-to-center separations, $d$. The FRET efficiency between monolayers of donor and acceptor QDs in bilayer structures has been previously shown to be well described by

$$
E_{F R E T}=\left[1+2 d^{4} / c_{A c c} \pi R_{0}^{6}\right]^{-1}
$$

where $d$ is the donor-acceptor center-to-center distance, $R_{0}$ is the Förster radius and $C_{A c c}$ is the acceptor concentration. ${ }^{33-35}$ Using the same acceptor concentration as for the trilayer system, the FRET efficiency for the donor-acceptor bilayers is calculated using a Förster radius, $R_{0}=4.2 \mathrm{~nm}$. A Förster radius $R_{0}=(4.2 \pm 0.4) \mathrm{nm}$ was calculated from the spectral overlap of the donor emission and acceptor absorption for this donor-acceptor pair, using a volume weight refractive index $n=1.5 \pm 0.3$ and the measured quantum yield of the donor QD monolayer of 3\%. ${ }^{15,35}$

The interaction between monolayers of CdTe QDs and $5.5 \mathrm{~nm}$ diameter $\mathrm{Au}$ NPs has been the subject of a previous separate study, reported in reference 15. It was previous shown that the dependence of the NRET efficiency on both the separation between the QDs and Au NPs, or the Au NP concentration, in a QD-Au NP bilayer 
structure, could be described using a NSET formalism. The NSET efficiency for energy transfer from the QDs to a plane of MNP acceptors is given by

$$
E_{\mathrm{NSET}}=\left[1+\frac{3}{c_{A u} \pi d_{0}{ }^{4}} \cdot \frac{\left(d-r_{A u}\right)^{3}}{\left(3 d-r_{A u}\right)}\right]^{-1}
$$

where $d_{0}$ is the characteristic distance at which the energy transfer efficiency is $50 \%$ for a single emitter-MNP pair. In this case the MNP is the acceptor and $d$ is the donor-acceptor center-to-center distance between the QD monolayer and Au NP monolayer. $E_{\text {NSET }}$ is calculated using a $\mathrm{Au}$ NP concentration of $c_{A u}=(0.12 \pm 0.01) \times 10^{17} \mathrm{~m}^{-2}$ (corresponding to the trilayer samples) and $d_{0}=3.1 \mathrm{~nm}$ (determined from the reference donor-Au NP bilayer samples). Comparison of the three cases, in Figure 3, shows clearly the large enhancement of the energy transfer range via LSP coupled FRET.

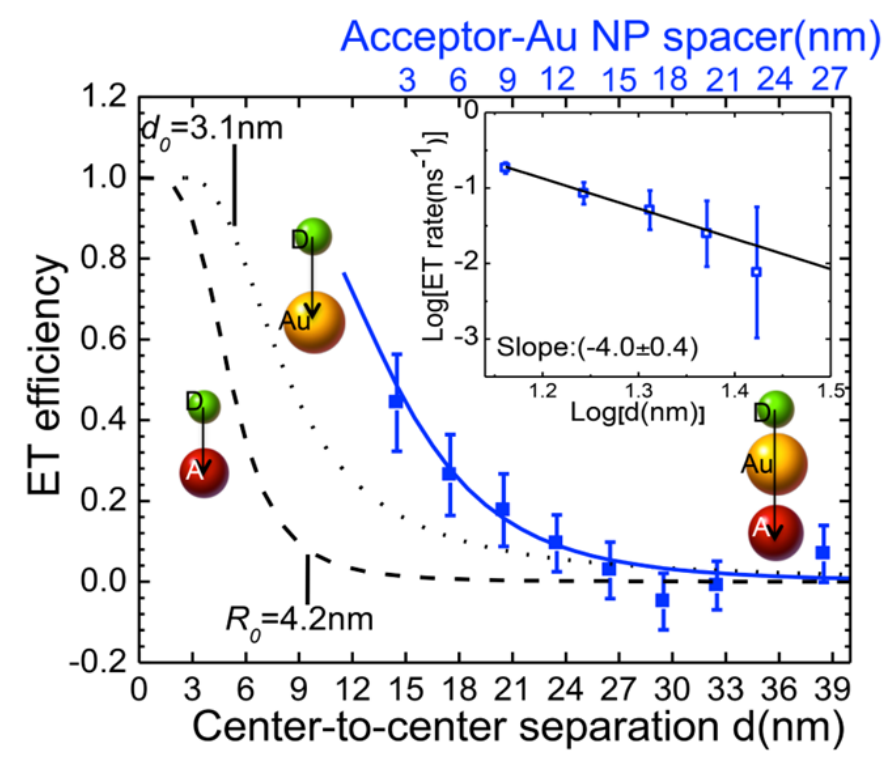

Figure 3. The LSP coupled FRET efficiency in trilayer structures (blue solid squares) with varying acceptor-Au NP spacer thickness, $t_{A c c-A u}$, (top axis) and a fixed donor- 
Au NP spacer thickness, $t_{A c c-A u}=3 \mathrm{~nm}$. All samples have $c_{A c c}=(0.8 \pm 0.1) \times 10^{17} \mathrm{~m}^{-2}$, $C_{\text {Don }}=(1.8 \pm 0.2) \times 10^{17} \mathrm{~m}^{-2}$ and $c_{A u}=(0.12 \pm 0.01) \times 10^{17} \mathrm{~m}^{-2}$. The corresponding donor-acceptor center-to-center separation, $d$, is shown on the bottom axis. The blue solid line is the numerical simulation of the LSP coupled FRET using the Green's tensor formalism (equation 1). The conventional FRET efficiency expected for this donor-acceptor pair is also shown (black dashed line calculated using equation 2), with the same acceptor monolayer concentration and $R_{0}=4.2 \mathrm{~nm}$. The NRET efficiency for the donor-Au NP bilayer based on the NSET formalism, with $d_{0}=3.1 \mathrm{~nm}$, is also included for comparison (black dotted line calculated using equation 3). The inset shows the dependence of the LSP coupled energy transfer rate on the separation, $d$.

Using the Green's tensor approach described earlier, we calculated numerically the LSP coupled energy transfer efficiency for the fixed donor-Au NP separation and varying acceptor-Au NP separation. The results of the numerical simulation are shown as the solid line in Figure 3. As before the theoretical results are in good agreement with the trilayer LSP coupled FRET efficiency experimental data. The agreement of the numerical simulation with experimental data, shown in both figures 2(b) and 3, validates the model. Further simulation results will be presented later.

Analysis of the experimentally measured separation dependence of the LSP coupled energy transfer rate as a function of the donor-acceptor center-to-center distance, $d$, shows a $d^{-(4.0 \pm 0.4)}$ dependence, see inset of Figure 3. As seen earlier, conventional FRET between separated planes of donor and acceptor QDs exhibits a 
$d^{-4}$ dependence. ${ }^{33-35}$ It is found that the expression $E_{F R E T}=\left[1+2 d^{4} / c_{A c c} \pi R_{0}^{6}\right]^{-1}$ fits the experimental data plotted in Figure 3 for $R_{0}=(8.1 \pm 0.7) \mathrm{nm}$, which can be considered as the LSP enhanced effective Förster radius. The fit is shown in the Supplementary Information, Figure $\mathrm{S} 2$. The enhanced $R_{0}$ corresponds to a $\sim 2$ fold increase in the Förster radius in the trilayer structures compared with the donoracceptor bilayer. The observation of the $d^{-4}$ separation dependence for the LSP coupled FRET indicates that the LSPs can enhance the dipole-dipole coupling while maintaining the conventional FRET-like separation dependence.

To better elucidate the fundamental donor-Au NP-acceptor interaction, we next investigate numerically the energy transfer efficiency for a single donor-Au NPacceptor triad. The donor QD and acceptor QD are placed on opposite sides of the Au NP and have the same properties as in the trilayer samples. The numerical results for the donor-acceptor energy transfer efficiency, $E_{L S P-F R E T}$, as a function of the donoracceptor center-to-center distance, $d$, are shown in Figure 4. Two cases are considered, firstly, the donor-Au NP separation, $t_{D o n-A u}$, is kept fixed and the acceptor-Au NP separation, $t_{A c c-A u}$, is varied, and secondly, $t_{A c c-A u}$ is kept fixed with varying $t_{D o n-A u}$, shown in Figure 4(a) and Figure 4(b), respectively. In Figure 4(a) the numerical simulation results are shown for two values of $t_{D o n-A u}, 3 \mathrm{~nm}$ and $6 \mathrm{~nm}$. The case of a single donor-acceptor pair, in the absence of a Au NP is also shown as a reference. The lines shown in Figure 4(a) correspond to fitting the numerical simulation data with a conventional FRET model for the energy transfer efficiency for $\underline{\text { a single donor-acceptor pair, }}$ 


$$
E_{\text {FRET }}=\frac{1}{1+\frac{d^{n}}{R_{0}^{n}}} .
$$

The fitting shows that over one order of magnitude of the donor-acceptor center-tocenter separation, $d$, the Au NP-mediated donor-acceptor interaction closely resembles conventional FRET $(\mathrm{n}=6)$, but with an increased Förster radius, $R_{0}$. For the two donor-Au NP distances investigated, $t_{D o n-A u}=3 \mathrm{~nm}$ and $t_{D o n-A u}=6 \mathrm{~nm}$, the corresponding Förster radii are $R_{0}=8.2 \mathrm{~nm}$ and $R_{0}=6.8 \mathrm{~nm}$, respectively. Comparing this with the Förster radius of the donor-acceptor pair in the absence of the $\mathrm{Au} \mathrm{NP}, R_{0}=4.3 \mathrm{~nm}$, we see that the Förster radius can be nearly doubled, depending on the donor-Au NP separation, $t_{D o n-A u}$. As the donor is moved further from the $\mathrm{Au}$ NP, the enhancement of the Förster radius becomes less and less prominent, eventually vanishing. It can be noted that the Förster radius of $4.3 \mathrm{~nm}$ obtained for the donor-acceptor pair is in agreement the experimentally determined Förster radius $R_{0}=(4.2 \pm 0.4)$ nmwhich was discussed earlier.
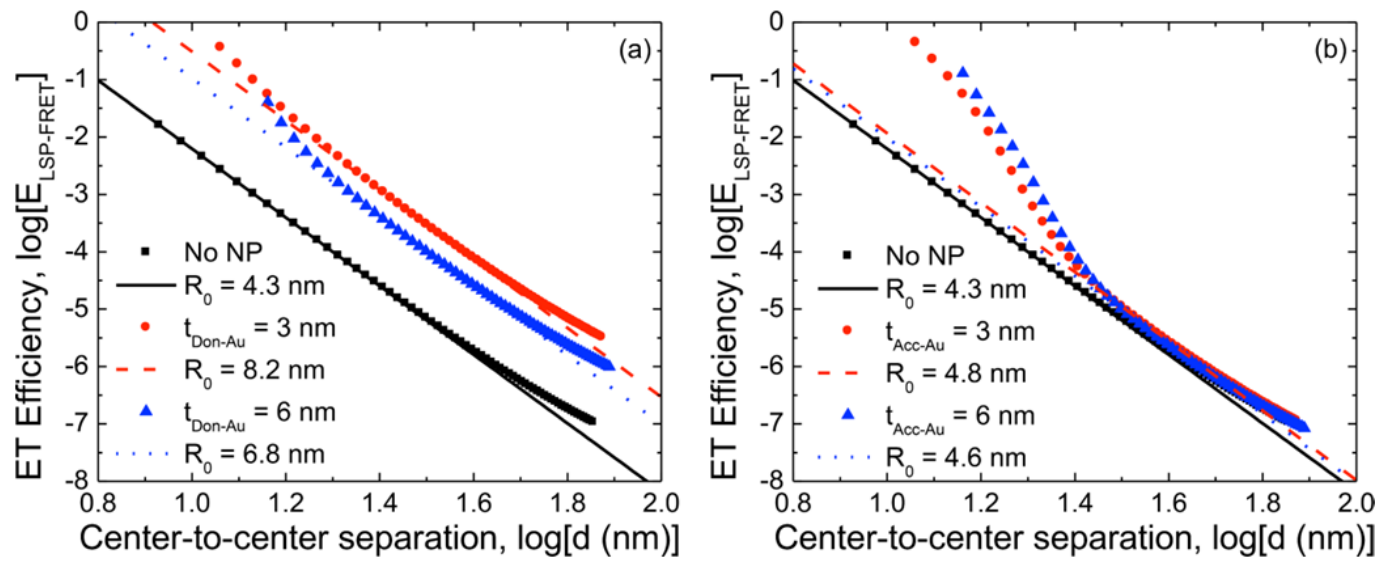

Figure 4. (a) Calculated LSP coupled energy transfer efficiency, $E_{L S P-F R E T}$, as a function 
of the donor-acceptor center-to-center separation, $d$, with fixed donor-Au NP separations $t_{D o n-A u}=3 \mathrm{~nm}$ (red circles) and $6 \mathrm{~nm}$ (blue triangles). The symbols represent the numerical simulation results calculated using the Green's tensor formalism. The lines represent the fit of these results with a FRET model given by equation 4. (b) Calculated LSP coupled energy transfer efficiency, $E_{\text {LSP-FRET }}$, as a function of the donor-acceptor center-to-center separation, $d$, for fixed acceptor-Au NP separations $t_{A c c-A u}=3 \mathrm{~nm}$ (red circles) and $6 \mathrm{~nm}$ (blue triangles). Both axes are logarithmic. The case of a single donoracceptor pair, without a Au NP is also shown (black circles) as a reference in both panels.

Figure 4(b) shows the same calculated energy transfer efficiency dependence on the donor-acceptor separation, $d$, but now it is the acceptor-Au NP spacer thickness, $t_{A c c-A u}$, which is kept fixed while the donor-Au NP spacer thickness, $t_{D o n-A u}$, increases. Two values of $t_{A c c-A u}$ are considered, $3 \mathrm{~nm}$ and $6 \mathrm{~nm}$. In this case it is seen that the FRET model is only valid over a much smaller range of $d$. Furthermore, within this range the fitting shows only a very small enhancement of the Förster radius from $R_{0}=$ $4.3 \mathrm{~nm}$ to $R_{0}=4.8 \mathrm{~nm}$ for $t_{A c c-A u}=3 \mathrm{~nm}$ and $R_{0}=4.6 \mathrm{~nm}$ for $t_{A c c-A u}=6 \mathrm{~nm}$. The increase in the LSP coupled FRET efficiency at the lower values of $d$ corresponds to where the donor and Au NP are sufficiently close that the LSP coupled FRET is once again strongly influenced by the donor-plasmon coupling. Comparison of Figures 4(a) and 4(b) shows that the donor-acceptor interaction in the presence of the Au NP is more sensitive to $t_{D o n-A u}$ than it is to $t_{A c c-A u}$. This is manifest in the greater sensitivity of the energy transfer efficiency, $E_{L S P-F R E T}$, to the change in $t_{D o n-A u}$ for a fixed $t_{A c c-A u}$ compared with modification of $E_{L S P-F R E T}$ for changing $t_{A c c-A u}$ with a constant $t_{D o n-A u}$. 
To further investigate the influence of the placement of the Au NPs on the LSP coupled energy transfer mechanism, we calculated the LSP coupled FRET efficiency between the donor and acceptor as a function of the position of an intermediate Au NP for a three values of donor-acceptor separation, $d$. The results are shown in Figure 5. To facilitate direct comparison of the effect of the position of the Au NP for different values of $d$, we plot the relative energy transfer efficiency, which is ratio of the energy transfer efficiency between the donor QD and acceptor QD in the presence of the Au NP, $E_{L S P-F R E T}$, and in the absence of the Au NP, $E_{F R E T}$. To plot the data for a number of different values of $d$ on the same graph, the intermediate Au NP position is presented as a fraction, $\quad x \quad$ This fraction is given by $x=t_{D o n-A u} /\left[d-\left(r_{D}+r_{A}+2 \times r_{A u}\right)\right]=t_{D o n-A u} /\left[t_{D o n-A u}+t_{A c c-A u}\right]$ and can vary from 0 to 1, where $x=0$ and $x=1$ correspond to the Au NP adjacent to the donor and acceptor, respectively.

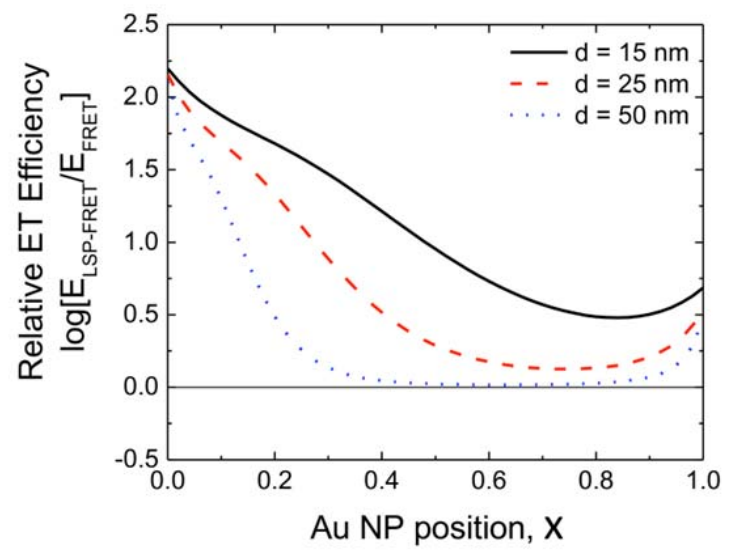

Figure 5. Relative energy transfer efficiency as a function of the intermediate Au NP position between the donor and acceptor QDs, for several fixed donor-acceptor centerto-center distances, $d$. 
It is clearly evident from Figure 5 that the LSP coupled FRET efficiency is more enhanced when the Au NP is close to the donor $(x \sim 0)$ rather than when it is close to the acceptor $(x \sim 1)$. Furthermore, the LSP coupled FRET efficiency with the $\mathrm{Au}$ NP close to the donor extends further than for Au NP closer to the acceptor. For smaller values of $d$ there evidence of QD-plasmon coupling for all positions of the $\mathrm{Au}$ $\mathrm{NP}$, as demonstrated by the fact that the logarithm of the relative energy transfer efficiency does not go to zero, i.e. the LSP coupled energy transfer efficiency is enhanced compared with its value in the absence of the Au NP. For $d=50 \mathrm{~nm}$, the Au NP can be placed sufficiently far from both the donor and acceptor QDs such that neither QD is coupled to the Au NP, and the energy transfer efficiency is the same as in the absence of the Au NP.

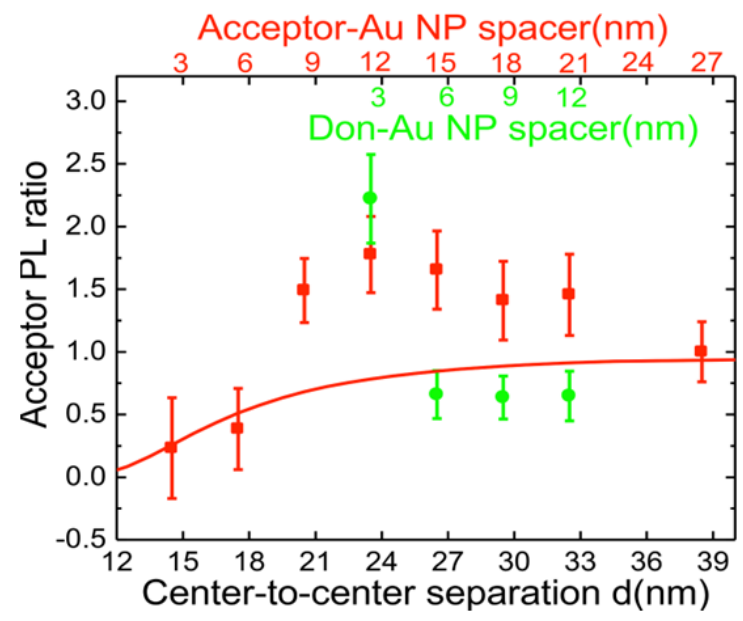

Figure 6. The acceptor PL ratio, $I_{A c c, T r i} / I_{A c c, B L}$, in trilayer structures, as a function of the donor-Au NP spacer thickness, $t_{A c c-A u}$ (top axis, inner values), acceptor-Au NP spacer thickness, $t_{A c c-A u}$ (top axis, outer values) and donor-acceptor center-to-center distance, $d$ (bottom axis). The samples with a fixed acceptor-Au NP separation, 
$t_{A c c-A u}=12 \mathrm{~nm}$, and varying $t_{D o n-A u}$ with $C_{A u}=(0.13 \pm 0.02) \times 10^{17} \mathrm{~m}^{-2}$ are shown as green circles. The samples with a fixed donor-Au NP separation, $t_{D o n-A u}=3 \mathrm{~nm}$, and varying $t_{A c c-A u}$ with $c_{A u}=(0.12 \pm 0.01) \times 10^{17} \mathrm{~m}^{-2}$ are shown as red squares. The solid line is the calculated acceptor PL ratio, $I_{A c c, A u} / I_{A c c}=1-E_{\text {NSET }}$, for acceptor-Au NP bilayers, with the same separations and Au NP concentration as the varying acceptor$\mathrm{Au}$ NP separation trilayer samples. This is calculated using equation 3.

Having shown the influence of the placement of the Au NP monolayer on the LSP coupled FRET efficiency, it is interesting to consider the influence of the various separations within the trilayer structure on the acceptor emission. The acceptor PL ratio, $I_{A c c, T r i} / I_{A c c, B L}$, as a function of varying acceptor-Au NP separation, with a fixed donor-Au NP separation, $t_{D o n-A u}=3 \mathrm{~nm}$, is shown in Figure 6. The PL ratio expected for acceptor-Au NP bilayers with varying QD-Au NP separation relative to the emission for an acceptor monolayer, $I_{A c c, A u} / I_{A c c}$, is also shown for comparison. This acceptor PL ratio is calculated based on the NSET formalism using $d_{0}=5.7 \mathrm{~nm}$ in equation 3. The value for $d_{0}$ was determined from experimental data for the acceptorAu NP reference samples. ${ }^{15}$ In the trilayer samples quenching of the acceptor PL is observed for the shortest acceptor-Au NP separations. This suggests that direct quenching of the acceptor PL by the Au NPs is dominant despite the highest LSP coupled FRET efficiency and fastest LSP coupled FRET rates at the smaller acceptor$\mathrm{Au}$ NP separations. At larger separations the acceptor PL in the trilayer structure increases, showing that the acceptor PL enhancement due to the LSP coupled FRET 
competes favorably with direct quenching of the acceptor emission by the Au NPs in this range. A maximum PL ratio of $\sim 1.8$ is observed for a $t_{A c c-A u}=12 \mathrm{~nm}$. As the separation is further increased, the acceptor PL ratio reduces as the LSP coupled FRET contribution decreases.

The PL ratio, for the varying donor-Au NP separation and varying acceptor$\mathrm{Au}$ NP separation, is also presented as a function of the donor-acceptor center-tocenter distance, $d$, on the bottom axis of Figure 6. The acceptor PL is more sensitive to the donor-Au NP separation, $t_{D o n-A u}$, than the acceptor-Au NP separation, $t_{A c c-A u}$. For the same donor-acceptor center-to-center distance, $d$, maximum enhancement of the acceptor PL requires the smallest donor-Au NP separation. These observations are in agreement with the earlier results which showed that that plasmon-donor coupling has a larger influence on LSP coupled FRET in this donor-Au NP-acceptor energy transfer system. The greater quenching of the acceptor PL in the trilayer structures with larger donor-Au NP separations, where the LSP coupled FRET efficiency is low and direct quenching by the Au NPs dominates, is due to the slightly higher Au NP concentration for that set of samples.

The influence of the interplay between the acceptor-Au NP separation and the $\mathrm{Au}$ NP concentration on the acceptor emission was also investigated. The acceptor PL ratio, $I_{A c c, T r i} / I_{A c c, B L}$, as a function of Au NP concentration is shown in Figure 7(a) for two different acceptor-Au NP separation, $t_{A c c-A u}=6 \mathrm{~nm}$ and $12 \mathrm{~nm}$, respectively. The donor-Au NP separation is fixed at $3 \mathrm{~nm}$. Also shown for comparison is the measured PL ratio for acceptor-Au NP bilayer structures relative to the emission from an acceptor monolayer, $I_{A c c, A u} / I_{A c c}$, along with fits based on the NSET model with $d_{0}=5.7 \mathrm{~nm}$ (solid lines calculated using equation 3). For both $t_{A c c-A u}$ separations the 
acceptor PL in the trilayer structure shows a maximum at a specific concentration. At the larger Au NP concentrations the trend of the acceptor PL ratio in the trilayer structures is similar to that observed for the acceptor-Au NP bilayer structures. As previously reported, this is a result of the competition between direct quenching of the acceptor PL by the Au NPs and enhancement of the acceptor emission due to LSP coupled FRET. ${ }^{25}$ The Au NP concentration at which the maximum PL ratio occurs is higher for $t_{A c c-A u}=12 \mathrm{~nm}$ than for $t_{A c c-A u}=6 \mathrm{~nm}$. Additionally, the trilayer structure with $t_{A c c-A u}=6 \mathrm{~nm}$ shows greater sensitivity to the Au NP concentration in the form of a narrower peak in PL enhancement. The shift in the PL ratio peak to lower Au NP concentrations and the increased sensitivity is a consequence of the interplay between the enhancement of the acceptor PL due to LSP coupled FRET and the direct acceptor PL quenching. The quenching due to NRET from the QDs to the Au NPs is a function of both the acceptor-Au NP separation and the Au NP concentration. As seen earlier, in equation 3 , the NSET efficiency has the form $E_{\text {NSET }}=1 /\left(1+\right.$ const $\left.\cdot d^{2} / c_{A u}\right)$, and therefore, the increase in the quenching efficiency due to the reduction of the acceptor-Au NP separation from $12 \mathrm{~nm}$ to $6 \mathrm{~nm}$, can be compensated by reducing the $\mathrm{Au}$ NP concentration. Consequently, the maximum acceptor PL enhancement will occur at a lower Au NP concentration, as observed. 

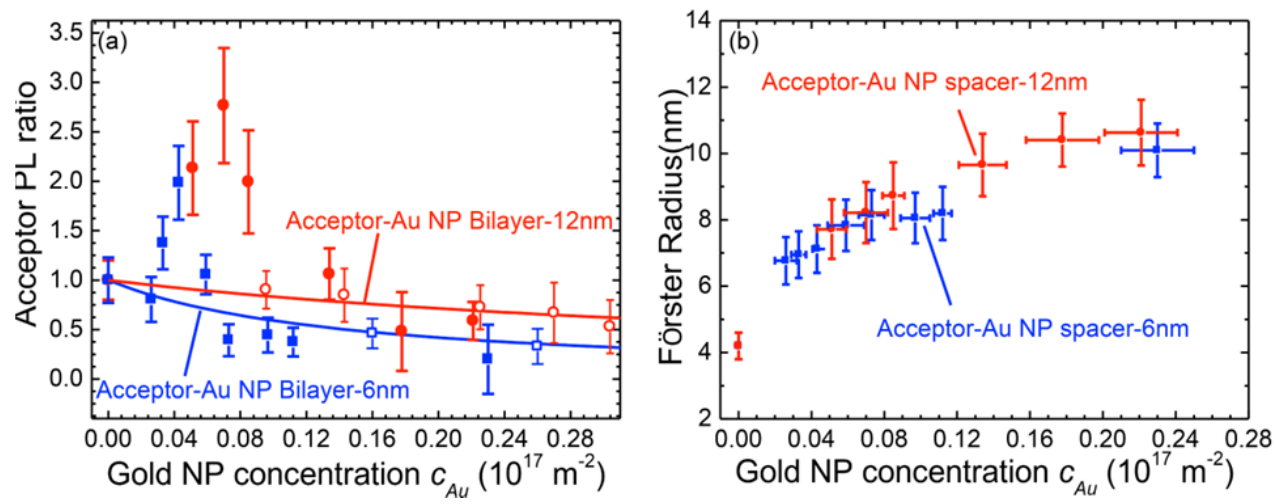

Figure 7. (a) PL ratio of the acceptor PL in a trilayer structure, $I_{A c c, T r i} / I_{A c c, B L}$, and the acceptor on Au NP structure, $I_{A c c, A u} / I_{A c c}$, as a function of the Au NP concentration. The trilayer samples with an acceptor-Au NP separation of $t_{A c c-A u}=6 \mathrm{~nm}$ (solid blue squares) and $t_{A c c-A u}=12 \mathrm{~nm}$ (solid red circles) have a fixed donor-Au NP separation, $t_{D o n-A u}=3 \mathrm{~nm}$. Data for the acceptor-Au NP bilayer samples with an acceptor-Au NP separation of $t_{A c c-A u}=6 \mathrm{~nm}$ (open blue squares) and $t_{A c c-A u}=12 \mathrm{~nm}$ (open red circles) are also shown. The solid lines show the calculated acceptor PL ratio for acceptor-Au NP bilayers with the same separations using the NSET model (equation 3). (b) The dependence of the extracted effective Förster radius for trilayer samples on the $\mathrm{Au}$ NP concentration. The acceptor-Au NP separations are $t_{A c c-A u}=6 \mathrm{~nm}$ (solid blue squares) and $t_{A c c-A u}=12 \mathrm{~nm}$ (solid red circles), with a fixed donor-Au NP separation, $t_{D o n-A u}=3 \mathrm{~nm}$.

Using the same set of trilayer structures we can probe how the LSP coupled FRET characteristic distance depends on the Au NP concentration. From the donor PL decays of trilayer structures and donor-Au NP bilayer reference samples, the LSP coupled energy transfer efficiency, $E_{L S P-F R E T}=1-\tau_{\text {Don,Tri }} / \tau_{\text {Don,Au }}$ is determined. 
Using the expression for FRET to a plane of acceptors described earlier in equation 2, $E_{F R E T}=\left[1+2 d^{4} / c_{A c c} \pi R_{0}^{6}\right]^{-1}$ where $d$ is the donor-acceptor center-to-center distance and $c_{A c c}$ is the experimentally measured concentration in the acceptor QD monolayer for each sample, the effective Förster radius, $R_{0}$, for the LSP coupled system is extracted at each Au NP concentration. These data are presented in Figure 7(b). The effective Förster radius increases with increasing Au NP concentration, and values as high as $10 \mathrm{~nm}$ are determined at the largest Au NP concentrations investigated. The conventional Förster radius, $R_{0}=(4.2 \pm 0.4) \mathrm{nm}$ for the donor-acceptor bilayer reference is shown at zero Au NP concentration. It is observed that for fixed donor$\mathrm{Au}$ NP separation, $t_{D o n-A u}$, the dependence of the Förster radius on the Au NP concentration appears to be independent of the acceptor-Au NP separation, $t_{A c c-A u}$. This suggests that the donor-plasmon coupling is the dominant influence on the LSP coupled FRET efficiency. This observation also agrees with the theoretical results presented in Figure 4(a). The numerical simulation results in Figure 3 showed that under certain conditions the LSP coupled FRET efficiency follows a $d^{-4}$ separation dependence with a single value for the effective $R_{0}$. The trilayer structures with varying acceptor-Au NP separation, presented in Figure 3, had a Au NP concentration of $c_{A u}=(0.12 \pm 0.01) \times 10^{17} \mathrm{~m}^{-2}$. This concentration corresponds to a Förster radius of $9 \pm 1 \mathrm{~nm}$ from Figure 7(b). This value agrees, within the error range, with the Förster radius obtain from fitting directly the donor-acceptor separation dependence of the LSP coupled FRET efficiency in Figure 3 using equation 2. 


\section{CONCLUSIONS}

It has been demonstrated that the signatures of LSP coupled FRET between donor and acceptor QD planes depend strongly on the position of an intermediate Au NP monolayer. The dependence of the LSP coupled FRET efficiency on the separations between the three components of the system has been theoretically and experimentally investigated. The largest LSP coupled FRET rates and efficiencies are observed for the smallest donor-Au NP and acceptor-Au NP separations. However, LSP coupled FRET through the intermediate Au NP layer is observed to be more sensitive to the donor-Au NP separation. It was experimentally observed that the dependence of the LSP coupled FRET efficiency on the donor-acceptor separation had the same $d^{-4}$ form as conventional FRET. The independence of the Förster radius on the acceptor-Au NP separation indicates a LSP coupled FRET system in which donor-plasmon coupling is dominant. The dipole-dipole behaviour of the separation dependence of the LSP coupled FRET efficiency suggests that the strongly coupled donor-plasmon system can be considered as an enhanced donor dipole. Theoretical studies have confirmed that donor-plasmon coupling provides greater enhancement of the LSP coupled FRET efficiency and range compared with acceptor-plasmon coupling in the system studied. Additionally, the numerical simulations confirm that LSP coupled FRET is well described a $d^{-4}$ distance dependence with an enhanced Förster radius over particular ranges of the donor-acceptor separation. The possibility to engineer systems with a well-defined distance dependence has potential as a longrange spectroscopic ruler or for sensing applications. The characteristic energy transfer distance can be tuned though the Au NP concentration. For any acceptor-Au NP separation the Au NP concentration can be adjusted to optimise the LSP coupled 
FRET enhanced acceptor PL. The large enhancement of the acceptor emission reported, despite competition with direct quenching by the Au NPs, demonstrates that LSP coupled FRET could be suitable for light emitting device application.

\section{METHODS}

The bilayer and trilayer structures were prepared by a layer-by-layer technique. They were fabricated on quartz substrates covered with a polyelectrolyte buffer layer. Bilayer structures comprised of a monolayer of QDs and a monolayer of $\mathrm{Au}$ NPs separated by a PE spacer layer. Trilayer structures comprised of donor and acceptor QD monolayers, separated from an intermediate Au NP layer by PE spacer layers. The PE spacer layers are formed using bilayers of oppositely charged polyelectrolytes, namely, positively charged poly(diallyldimethylammonium chloride) (PDDA) and negatively charged poly(sodium 4-styrene sulfonate) (PSS). Further details on the sample preparation and the solution concentrations can be found in references 27 and 35. The PE layer thicknesses were validated by X-ray diffraction measurements. PE spacer layers have been previously used to investigate the distance dependence of FRET between QDs and QD-plasmon coupling effects. ${ }^{33-37}$

The negatively charged CdTe QDs, stabilized by thioglycolic acid, were synthesized in water according to standard procedures. ${ }^{38,39}$ The sizes of the QDs and the QD concentrations in the multilayer structures were determined from the absorption spectra. ${ }^{38}$ The quantum yield (QY) of the QDs in the layers was determined in comparison to the luminescent standard Rhodamine 6G. The measured QY in the monolayers have been validated by comparison of the theoretical and experimentally measured separation and concentration dependencies of FRET 
between donor and acceptor nanocrystal QDs. The theoretical dependencies were calculated using the measured QY of the QDs in monolayers. ${ }^{35}$

The colloidal Au NPs were stabilized by 4-dimethylaminopyridine (DMAP) and had an average diameter of $5.5 \mathrm{~nm} .{ }^{40}$ To achieve different Au NP concentrations in the layer the immersion time in the Au NP solution, with a concentration of the order of $1 \times 10^{-7} \mathrm{M}$, was varied between 1 and 20 minutes. The fitting of the solution spectra $^{41,42}$ gave a molar extinction coefficient of $4.19 \times 10^{6} \mathrm{M}^{-1} \mathrm{~cm}^{-1}$ at the wavelength of the LSP absorption peak, which was used to estimate the gold NP concentration from the Au NP layer absorption spectra.

Absorption spectra were measured using a double beam UV-vis spectrometer (Shimadzu UV-2401 PC). Steady-state PL spectra were recorded using a PerkinElmer LS S5 fluorescence spectrometer with a $400 \mathrm{~nm}$ excitation wavelength, provided by a pulsed Xenon lamp. The donor QD PL decays were recorded over an area of $80 \times 80 \mu \mathrm{m}^{2}(150 \times 150$ pixels $)$ using a PicoQuant Microtime200 time-resolved confocal microscope system with 150 ps resolution. Picosecond excitation pulses, at a wavelength of $470 \mathrm{~nm}$, were provided by a LDH-480 laser head controlled by a PDL-800B driver (PicoQuant). A $550 \mathrm{~nm}$ broadband filter with a full-width-halfmaximum of approximately $(70 \pm 5) \mathrm{nm}$ was used to select the donor QD emission. Typically, a repetition rate of $10 \mathrm{MHz}$ and an integration time of $4 \mathrm{~ms}$ per pixel were used. The PL decays can be fitted using two exponentials and the average decay lifetime, $\tau$, is calculated from the intensity weighted mean. All samples were fabricated and measured at room temperature. 
Conflict of Interest: The authors declare no competing financial interest.

\section{AUTHOR INFORMATION}

Corresponding Author

*E-mail: bradlel@tcd.ie.

Present Address

$\dagger$ Now at Philips Research Laboratories, 5656 AE Eindhoven, The Netherlands

\section{ACKNOWLEDGMENTS}

We thank Robert Gunning for the thickness measurements of the polyelectrolyte layers by X-ray diffraction. This work was financially supported by Science Foundation Ireland projects 10/IN.1/12975 and 07/IN.1/I1862, and by a grant from the Research Grant Council of the Hong Kong S.A.R., China (project No. [T23713/11]). NG, VL and AR acknowledge a partial financial support from the EU FP7 Network of Excellence "Nanophotonics4Energy".

\section{ASSOCIATED CONTENT}

Supporting Information. Photoluminescence spectra for a trilayer structure as a function of the donor QD-Au NP layer separation. Center-to-center separation dependence of the LPS coupled energy transfer efficiency. This material is available free of charge via the Internet at http://pubs.acs.org. 


\section{REFERENCES}

(1) Förster, T. Intermolecular Energy Migration and Fluorescence. Annalen der Physik 1948, 2, 55-75.

(2) Stryer, L.; Haugland, R. P. Energy Transfer - A Spectroscopic Ruler. Proc. Natl. Acad. Sci. 1967, 58, 719-726.

(3) Kasprzak, A. A. The Use of FRET in the Analysis of Motor Protein Structure. Methods Mol. Biol. 2007, 392,183.

(4) Iqbal, A.; Wang, L.; Thompson K. C.; Lilley, D M. J; Norman, D. G. The Structure of Cyanine 5 Terminally Attached to Double-Stranded DNA: Implications for FRET Studies, Biochemistry 2008, 47, 7857-7862.

(5) Yun, C. S.; Javier, A.; Jennings, T.; Fisher, M.; Hira, S.; Peterson, S.; Hopkins, B.; Reich, N. O.; Strouse, G. F. Nanometal Surface Energy Transfer in Optical Rulers, Breaking the FRET Barrier. J. Am. Chem. Soc. 2005, 127, 3115-3119.

(6) Zhang, J.; Fu, Y.; Lakowicz, J. R. Enhanced Förster Resonance Energy Transfer (FRET) on a Single Metal Particle. J. Phys. Chem. C 2007, 111, 50-56.

(7) Anger, P.; Bharadwaj, P.; Novotny, L. Enhancement and Quenching of SingleMolecule Fluorescence. Phys. Rev. Lett. 2006, 96, 113002.

(8) Kühn, S.; Håkanson, U.; Rogobete, L.; Sandoghdar, V. Enhancement of SingleMolecule Fluorescence Using a Gold Nanoparticle as an Optical Nanoantenna. Phys. Rev. Lett. 2006, 97, 017402.

(9) Dulkeith, E.; Morteani, A. C.; Niedereichholz, T.; Klar, T. A.; Feldmann, J.; Levi, S. A.; van Veggel, F. C. J.M.; Reinhoudt, D. N.; Möller, M.; Gittins, D. Fluorescence Quenching of Dye Molecules near Gold Nanoparticles: Radiative and Nonradiative Effects. Phys. Rev. Lett. 2002, 89, 203002. 
(10) Pompa, P. P.; Martiradonna, L.; Della Torre, A.; Della Sala, F.; Manna, L.; de Vittorio, M.; Calabi, F.; Cingolani, R.; Rinaldi, R. Metal-Enhanced Fluorescence of Colloidal Nanocrystals with Nanoscale Control. Nat. Nanotechnol. 2006, 1, 126-130.

(11) Komarala, V. K.; Rakovich, Y. P.; Bradley, A. L.; Byrne, S. J.; Gun'ko, Y. K.; Gaponik, N.; Eychmüller, A. Off-resonance surface plasmon enhanced spontaneous emission from CdTe quantum dots. Appl. Phys. Lett. 2006, 89, 253118.

(12) Ozel, T.; Nizamoglu, S.; Sefunc, M. A.; Samarskaya, O.; Ozel, I. O.; Mutugun, E.; Lesnyak, V.; Gaponik, N.; Eychmüller, A.; Gaponenko, S. V. et al. Anisotropic Emission from Multi-Layered Plasmon Resonator Nanocomposites of Isotropic Semicondcutor Quantum Dots. ACS Nano 2011, 5, 1328-1334.

(13) Jennings, T. L.; Singh, M. P.; Strouse, G. F. Flourescent Lifetime Quenching Near d=1.5 nm Gold Nanoparticles. J. Am. Chem. Soc. 2006, 128, 5462-5467.

(14) Pons, T.; Medintz, I. L.; Sapsford, K. E.; Higashiya, S.; Grimes, A. F.; English, D. S.; Mattoussi, H. On the Quenching of Semiconductor Quantum Dot Photoluminescence by Proximal Gold Nanoparticles. Nano Lett. 2007, 7, 3157-3164.

(15) Zhang, X.; Marocico, C. A.; Lunz, M.; Gerard, V. A.; Gun'ko, Y. K.; Lesnyak, V.; Gaponik, N.; Susha, A. S.; Rogach, A. L.; Bradley, A. L. Wavelength, Concentration and Distance Dependence of Nonradiative Energy Transfer to a Plane of Gold Nanoparticles. ACS Nano 2012, 10, 9283-9290.

(16) Gersten, J. I.; Nitzan, A. Accelerated Energy Transfer Between Molecules Near a Solid Particle. Chem. Phys. Lett. 1984, 104, 31.

(17) Hua, X. M.; Gersten, J. I.; Nitzan, A. Theory of Energy Transfer Between Molecules Near Solid State Particles, J. Chem. Phys. 1985, 83, 3650-3659. 
(18) Govorov, A. O.; Lee, J.; Kotov, N. A. Theory of Plasmon-Enhanced Förster Energy Transfer in Optically Excited Semiconductor and Metal Nanoparticles, Phys. Rev. B 2007, 76, 125308.

(19) Wang, C. H.; Chen, C. W.; Chen, Y. T.; Wei, C. M.; Chen, Y. F.; Lai, C. W.; Ho, M. L.; Chou, P. T.; Hofmann, M. Surface Plasmon Enhanced Energy Transfer Between Type I CdSe/ZnS and Type II CdSe/ZnTe Quantum Dots, Appl. Phys. Lett. 2010, 96, 071906.

(20) Su, X. R.; Zhang, W.; Zhou, L.; Peng, X. N.; Pang, D. W.; Liu, S. D.; Zhou, Z. K.; Wang, Q. Q. Multipole-Plasmon-Enhanced Förster Energy Transfer Between Semiconductor Quantum Dots Via Dual-Resonance Nanoantenna Effects, Appl. Phys. Lett. 2010, 96, 043106.

(21) Faessler, V.; Hrelescu, C.; Lutich, A. A.; Osinkina, L.; Mayilo, S.; Jäckel, F.; Feldmann, J. Accelerating Fluorescence Resonance Energy Transfer with Plasmonic Nanoresonators, Chem. Phys. Lett. 2011, 508, 67-70.

(22) Zhao, X.; Wang, P.; Li, B. Surface Plasmon Enhanced Energy Transfer in MetalSemiconductor Hybrid Nanostructures. Nanoscale 2011, 3, 3056-3059.

(23) Lessard-Viger, M.; Rioux, M.; Rainville, L.; Boudreau, D. FRET Enhancement in Multilayer Core-Shell Nanoparticles. Nano Lett. 2009, 9, 3066-3071.

(24) L.-Viger, M.; Brouard, D., Boudreau, D. Plasmon-Enhanced Resonance Energy Transfer from a Conjugated Polymer to Fluorescent Multilayer Core-Shell Nanoparticles: A Photophysical Study. J. Phys. Chem. C 2011, 115, 2974-2981.

(25) Zhao, L.; Ming, T.; Shao, L.; Chen, H.; Wang, J. Plasmon-Controlled Förster Resonance Energy Transfer. J. Phys. Chem. C 2012, 116, 8287-8296. 
(26) Komarala, V. K.; Bradley, A. L.; Rakovich, Y. P.; Byrne, S. J.; Gun'ko, Y. K.; Rogach, A. L. Surface Plasmon Enhanced Förster Resonance Energy Transfer Between the CdTe Quantum Dots. Appl. Phys. Lett. 2008, 93, 123102.

(27) Lunz, M.; Gerard, V. A.; Byrne, S. J.; Gun'ko, Y. P.; Lesnyak, V.; Gaponik, N.; Susha, A. S.; Rogach, A. L.; Bradley, A. L. Surface Plasmon Enhanced Energy Transfer between Donor and Acceptor CdTe Nanocrystals Quantum Dot Monolayers. Nano Lett. 2011, 11, 3341-3345.

(28) West, R. G.; Sadeghi, S. M.; Enhancement of Energy Transfer between Qauntum Dots: The Impact of Metallic Nanoparticle Sizes. J. Phys. Chem. C 2012, 116, 2049620505.

(29) Kim, K.-S.; Kim, J.-H.; Kim, H., Laquai, F. Arifin, E., Lee, J.-K.; Yoo, S. I.; Sohn, B.-H. Switching Off FRET in the Hybrid Assemblies of Diblock Copolymer Micelles, Quantum Dots and Dyes by Plasmonic Nanoparticles. ACS Nano 2012, 6, 5051-5059.

(30) Lunz, M.; Zhang, X.; Gerard, V. A.; Byrne, S. J.; Gun'ko, Y. P.; Lesnyak, V.; Gaponik, N.; Susha, A. S.; Rogach, A. L.; Bradley, A. L. Effect of Metal Nanoparticle Concentration on Localised Surface Plasmon Mediated Förster Resonant Energy Transfer. J. Phys. Chem. C 2012, 116, 26529-26534.

(31) Ozel, T.; Martinez, P. L. H,; Mutlugun, E.; Akin, O.; Nizamoglu, S.; Ozel, I. O.; Zhang, Q.; Xiong, Q.; Demir, H. V.; Observation of Selective Plasmon-Exciton Coupling in Nonradiative Energy Transfer: Donor-Selective Vs. Acceptor-Selective Plexcitons. Nano Lett. 2013, 13, 3065-3072.

(32) Rogach, A. L.; Koktysh, D. S.; Harrison, M.; Kotov, N. A. Layer-by-Layer Assembled Films of HgTe Nanocrystals with Strong Infrared Emission. Chem. Mater. 2000, 12, 1526-1528. 
(33) Kim, D. G.; Okahara, S.; Nakayama, M.; Shim, Y. G. Experimental Verification of Förster Energy Transfer Between Semiconductor Quantum Dots. Phys. Rev. B 2008, 78, 153301.

(34) Lunz, M.; Bradley, A. L.; Chen, W.-Y. ; Gun’ko, Y. K. Förster Resonant Energy Transfer in Quantum Dot Layers. Superlattices Microstruct. 2010, 47, 98.

(35) Lunz, M.; Bradley, A. L.; Gerard, V. A.; Byrne, S. J.; Gun'ko, Y. P.; Lesnyak, V.; Gaponik, N. Concentration Dependence of Förster Resonant Energy Transfer Between Donor and Acceptor Nanocrystal Quantum Dot Layers: Effect of DonorDonor Interactions. Phys. Rev. B 2011, 83, 115423.

(36) Kulakovich, O.; Strekal, N.; Yaroshevich, A.; Maskevich, S.; Gaponenko, S.; Nabiev, I.; Woggon, U.; Artemyev, M. Enhanced Luminescence of CdSe Quantum Dots on Gold Colloids. Nano Lett. 2002, 2, 1449-1452.

(37) Chan, Y.-H.; Chen, J.; Wark, S. E.; Skiles, S. L.; Son, D. H.; Batteas, J. D. Using Patterned Arrays of Metal Nanoparticles to Probe Plasmon Enhanced Luminescence of CdSe Quantum Dots. ACS Nano 2009, 3, 1735-1744.

(38) Rogach, A. L.; Franzl, T.; Klar, T. A.; Feldmann, J.; Gaponik, N.; Lesnyak, V.; Shavel, A.; Eychmüller, A.; Rakovich, Y. P.; Donegan, J. F. Aqueous Synthesis of Thiol-Capped CdTe Nanocrystals: State-of-the-Art. J. Phys.Chem. C 2007, 111, 14628-14637.

(39) Lesnyak, V.; Gaponik, N.; Eychmüller, A. Colloidal Semiconductor Nanocrystals: The Aqueous Approach. Chem. Soc. Rev. 2013, 42, 2905-2929.

(40) Gittins, D. I.; Caruso, F. Spontaneous Phase Transfer of Nanoparticulate Metals from Organic to Aqueous Media. Angew. Chem. - Int. Ed. 2001, 40, 3001-3004.

(41) Jain, P. K.; Lee, K. S.; El-Sayed, I. H.; El-Sayed, M. A. Calculated Absorption and Scattering Properties of Gold Nanoparticles of Different Size, Shape and 
Composition: Applications in Biological Imaging and Biomedicine. J. Phys. Chem. B 2006, 110, 7238-7248.

(42) Amendola, V.; Meneghetti, M. Size Evaluation of Gold Nanoparticles by UVVis Spectroscopy. J. Phys. Chem. C 2009, 113, 4277-4285.

(43) Shevchenko, E. V.; Ringler, M.; Schwemer, A.; Talapin, D. V.; Klar, T. A.; Rogach, A. L.; Feldmann, J.; Alivisatos, A. P. Self-Assembled Binary Superlattices of CdSe and Au Nanocrystals and Their Fluorescence Properties. J. Am. Chem. Soc. 2008, 130, 3274-3275.

(44) Dung, H. T.; Knöll, L.; Welsch, D.-G. Intermolecular Energy Transfer in the Presence of Dispersing and Absorbing Media. Phys. Rev. A 2002, 65, 043813.

(45) Xu, Y. Electromagnetic Scattering by an Aggregate of Spheres. Appl. Opt. 1995, 34, 4573.

(46) Mackowski, D. W. Analysis of Radiative Scattering for Multiple Sphere Configurations. Proc. R. Soc. A Math. Phys. Eng. Sci. 1991, 433, 599-614.

(47) Chern, R.-L.; Liu, X.-X.; Chang, C.-C. Particle Plasmons of Metal Nanospheres: Application of Multiple Scattering Approach. Phys. Rev. E 2007, 76, 016609.

(48) L. Novotny, B. Hecht, Principles of Nano-Optics (CUP, Cambridge, UK, 2012).

(49) R. Ruppin, Decay of an Excited Molecule Near a Small Metal Sphere, J. Chem. Phys. 1982, 76, 1681. 


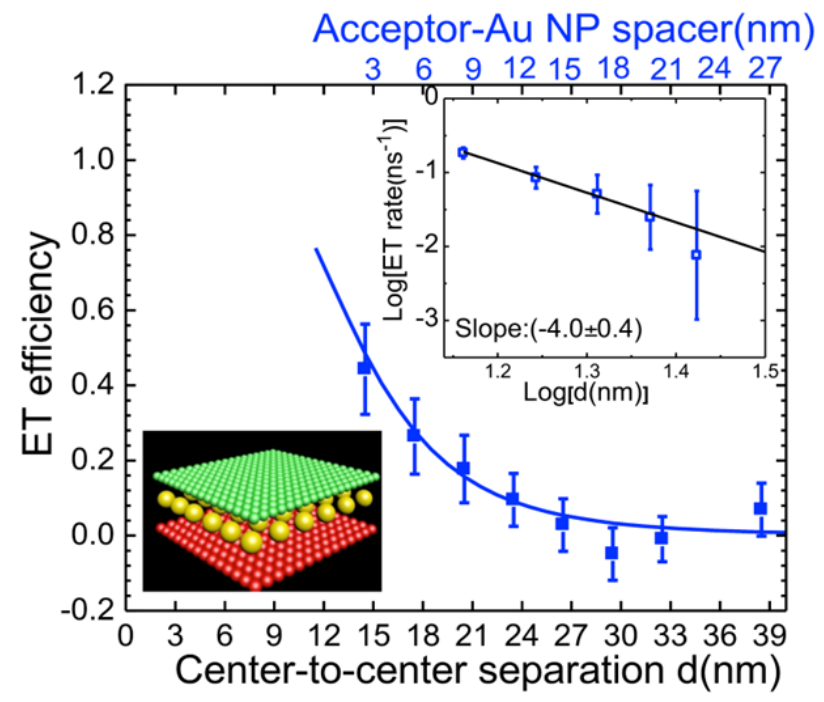

